\title{
Produção de cultivares de abobrinha italiana a pleno sol e sombreada no Nordeste brasileiro ${ }^{1}$
}

\author{
Antonia Mirian Nogueira de Moura Guerra², Maria Gabriela Magalhães Silva², Régila Santos \\ Evangelista $^{2}$, Edeilton Borges dos Santos ${ }^{2}$, Weber Pereira Nogueira ${ }^{2}$
}

\begin{abstract}
Resumo: A abobrinha italiana está entre as dez hortaliças de maior valor econômico e produção nacional. Entretanto, estudos de cultivares e seu cultivo sob sombreamento, são escassos. Sendo assim, esta pesquisa foi desenvolvida com o objetivo de avaliar o comportamento produtivo de cultivares de abobrinha a pleno sol e sombreada. Utilizou-se o delineamento de blocos, com quatro repetições e os tratamentos obedeceram ao esquema fatorial 5 × 2 sendo cinco cultivares de abobrinha ("Anita F1, Brenda F1, Irit F1, Corona F1 e Caserta") e dois sistemas de cultivos (a pleno sol e sombreamento com tela preta), respectivamente. O ambiente de cultivo influenciou a produção de abobrinhas italianas. Frutos com maior diâmetro foram produzidos pela cultivar Brenda F1. As cultivares Anita F1 e Corona F1 produziram frutos com maior comprimento e, Brenda F1 e Corona F1 frutos com maior massa. $\mathrm{O}$ cultivo a pleno sol proporcionou maior número de frutos por planta, com exceção da cultivar Irit F1 que apresentou maior número de frutos no cultivo sombreado. O cultivo a pleno sol apresentou maior produtividade e produção comercial quando comparado com o cultivo sombreado. A cultivar Brenda F1 cultivada a pleno sol apresentou maior produtividade e produção comercial. As cultivares Corona F1 e Irit F1 apresentaram maiores produtividade e produção comercial quando cultivada com sombreamento. $\mathrm{O}$ cultivo de abobrinha italiana sombreada nas condições da região Nordeste é viável, pois minimiza os efeitos negativos do ambiente de cultivo, evitando-se, principalmente frutos queimados pelo sol, assegurando sua qualidade visual, nutricional e sensorial.
\end{abstract}

Palavras-chave: Brenda F1; Caserta; Cucurbita pepo; Irit F1; Cultivares; Sistemas de cultivo.

\section{Production of italian zucchini cultivars at full sun and shaded in brazilian Northeast}

\begin{abstract}
Italian zucchini is among the ten vegetables of greatest economic value and national production. However, studies of cultivars and their cultivation under shade are scarce. Therefore, this research was developed with the objective of evaluating the productive behavior of zucchini cultivars under full sun and shade. A block design was used, with four replications and the treatments followed the $5 \times 2$ factorial scheme, five cultivars of zucchini ("Anita F1, Brenda F1, Irit F1, Corona F1 and Caserta") and two cultivation systems (a full sun and shading with black screen), respectively. The cultivation environment influenced the production of Italian zucchinis. Larger diameter fruits were produced by the cultivar Brenda F1. The cultivars Anita F1 and Corona F1 produced fruits with greater length and, Brenda F1 and Corona F1 fruits with greater mass. The cultivation in full sun provided a greater number of fruits per plant, with the exception of the cultivar Irit F1, which presented a greater number of fruits in the shaded cultivation. Full sun cultivation showed higher productivity and commercial production when compared to shaded cultivation. The cultivar Brenda F1 cultivated in full sun showed higher productivity and commercial production. The cultivars Corona F1 and Irit F1 showed higher productivity and commercial production when cultivated with shading. The cultivation of Italian zucchini shaded in the conditions of the Northeast region is feasible, since it minimizes the negative effects of the cultivation environment, avoiding, mainly fruits burned by the sun, ensuring its visual, nutritional and sensory quality.
\end{abstract}

Keywords: Brenda F1; Caserta; Cucurbita pepo; Irit F1; Cultivars; Cultivation systems.

\footnotetext{
${ }^{1}$ Submetido em 01/02/2020 e aprovado em 29/06/2020;

${ }^{2}$ Universidade Federal do Oeste da Bahia, Centro Multidisciplinar de Barra, Barra, BA, Brasil. E-mail: mirianagronoma@ hotmail.com (Autor correspondente) - ORCID: https://orcid.org/0000-0001-9475-306X; gabi2809@live.com - ORCID: https://orcid.org/0000-0001-5878-634X; regilasantos10@gmail.com, ORCID: https://orcid.org/0000-0001-5739-6382; edeilton17@gmail.com, ORICD: https://orcid.org/0000-00016002-5355; weberweber52@gmail.com, ORICD: https://orcid.org/0000-0002-6833-589X
}

Agropecuária Técnica, Areia-PB, v. 41, n. 1-2, p. 1-7, 2020

https://doi.org/10.25066/agrotec.v41i1-2.50535 


\section{Introdução}

A abobrinha italiana (Cucurbita pepo L.), integrante da família Cucurbitaceae, no Brasil é conhecida como abóbora de moita, abobrinha italiana ou abobrinha de tronco (Filgueira, 2013) e está entre as dez hortaliças de maior valor econômico e maior produção nacional. A produção brasileira de abobrinha em 2018 foi de 228.500 toneladas distribuídas entre 34.858 estabelecimentos agrícolas, sendo a região Sudeste responsável pela maior concentração de estabelecimentos $(48,00 \%)$ e produção nacional $(75,35 \%)$ (IBGE, 2019).

Apesar da importância alimentar e econômica da abobrinha, ainda é notável a maior exploração desta cucurbitácea na região Centro-Sul do Brasil, enquanto que as abóboras (Cucurbita moschata) e morangas (C. maxima) são mais exploradas na região Nordeste, fato este que leva a menor disponibilidade de estudos realizados em relação ao seu cultivo nas condições desta região, incluindo desempenho produtivo de cultivares e sua produção sob telas de sombreamento.

De fundamental importância para o cultivo da abobrinha é a recomendação de cultivares adequadas através da avaliação de genótipos, visto que materiais diferentes podem responder diferenciadamente quanto à produtividade (Filgueira, 2013). No Brasil, existe a necessidade de mais estudos quanto à adequação das cultivares, regiões e épocas de plantio de abobrinhas. $\mathrm{O}$ fato justifica-se porque, atualmente, existirem vários híbridos introduzidos no mercado que possuem precocidade em relação a vários atributos genéticos como, alta produtividade, uniformidade da produção e resistência à doenças em relação a cultivares pioneiras a exemplo da Cultivar Caserta.

As telas de sombreamento no interior do ambiente protegido podem proporcionar condição microclimática apropriada para o desenvolvimento da cultura, reduzindo os efeitos nocivos da alta taxa de incidência da radiação solar e da temperatura sobre a planta (Shahak et al., 2004). Essas telas de coberturas, devem apresentar boa ventilação para possibilitar o seu uso no verão, além de diminuir a temperatura e evitar danos no sistema radicular e foliar (Anglés, 2001).
Além das telas de sombreamento de cor preta, novas tecnologias na sua utilização, estão sendo empregadas com o objetivo principal de proteger as plantas da radiação solar direta. Esses materiais de polietileno de baixa densidade (PEBD) são de várias colorações (azul, vermelho, amarelo, cinza) com funções específicas na sua utilização. Por exemplo, a malha termo-refletora de alumínio, que promove boa ventilação, distribuição uniforme da luz e aporte máximo da luz difusa e da reflexão da radiação infravermelha, serve tanto para evitar o excesso da temperatura como para economizar energia (Huertas, 2006). As telas de coloração vermelha transferem mais a luz do espectro nas ondas vermelho e vermelho distante e difundem a luz que passa através da malha, sendo eficiente no desenvolvimento da planta; já as telas de coloração azul proporcionam luz do espectro em comprimento de onda de 440-490 nm, intensificando o fototropismo e a fotossíntese $(\mathrm{Li}$, 2006).

Também é possível com o cultivo em ambiente protegido obter aumento nos rendimentos, através do controle parcial de fatores responsáveis pelo crescimento e desenvolvimento das plantas, obtendo um produto de melhor qualidade para comercialização e reduzindo os efeitos da sazonalidade de produção (Guerra et al., 2017; Santi et al., 2013; Duarte e Peil, 2010; Queiroga et al., 2008).

Quando se trata de espécies como da família Cucurbitácea, de ciclo curto, clima quente e exigentes em luminosidade, o cultivo sob telas de sombreamento pode vir a interferir no crescimento e desenvolvimento das plantas. No entanto, a abobrinha tolera temperaturas medianas, na faixa de 10 a $30{ }^{\circ} \mathrm{C}$, e o excesso de calor e luminosidade podem comprometer a produção devido ao abortamento de flores e a qualidade dos frutos devido a queimaduras pelo sol (Madeira e Amaro, 2017).

Ao contrário, o cultivo sob tela de sombreamento apresenta-se como alternativa para proporcionar maior produção de frutos comercializáveis, de modo a permitir a exploração das plantas por maior período com possibilidade de maior produtividade por hectare.

Com o objetivo de obter referências que possam gerar mais informações que atendam às 
necessidades dos olericultores em melhorar o desempenho do cultivo da abobrinha italiana em regiões semi-áridas do Nordeste brasileiro, objetivou-se com esta pesquisa avaliar o comportamento produtivo de diferentes cultivares de abobrinha italiana submetidas a dois sistemas de cultivo, a pleno sol e sob tela preta de sombreamento.

\section{Material e Métodos}

A pesquisa foi conduzida em área experimental da Universidade Federal do Oeste da Bahia, Centro Multidisciplinar de Barra, município de Barra - BA (11 ${ }^{\circ} 5^{\prime} 23^{\prime \prime} \mathrm{S}, 43^{\circ} 8^{\prime} 30^{\prime \prime}$ $\mathrm{W}$, com uma altitude média de 398 metros acima do nível do mar). O clima da região segundo a classificação de Köeppen é do tipo Aw, que é um clima tropical com uma estação seca no tocante a irregularidades da precipitação pluviométrica, com período chuvoso compreendido entre os meses de dezembro a fevereiro, precipitação pluviométrica média de $335 \mathrm{~mm}$ para o trimestre e os demais meses praticamente secos, temperatura média do ar de $25,5{ }^{\circ} \mathrm{C}$, umidade relativa média do ar de $61 \%$. Durante o período do experimento, de abril a junho de 2018, as temperaturas máxima, mínima e média do ar foram de $32,5,18,5$ e $25,5^{\circ} \mathrm{C}$, respectivamente, com $0,0 \mathrm{~mm}$ de precipitação pluviométrica e umidade relativa do ar de 60,5\% (INMET, 2018).

A análise do solo da área experimental (0-20 cm) apresentou as características: $\mathrm{pH}$ em $\mathrm{H}_{2} \mathrm{O}=$ 5,5; $\mathrm{P}=3,0 \mathrm{mg} \mathrm{dm}^{-3}$ (Mehlich 1); $\mathrm{K}=46 \mathrm{mg} \mathrm{dm}^{-}$ 3; $\mathrm{Ca}^{2+}=0,9 \mathrm{cmol}_{\mathrm{c}} \mathrm{dm}^{-3} ; \mathrm{Mg}^{2+}=0,2 \mathrm{cmol}_{\mathrm{c}} \mathrm{dm}^{-3}$; $\mathrm{H}+\mathrm{Al}=3,3 \mathrm{cmol}_{\mathrm{c}} \mathrm{dm}^{-3}, \mathrm{~S}=1,55 \mathrm{cmol}_{\mathrm{c}} \mathrm{dm}^{-3}, \mathrm{~V}=$ $57 \%$ e M.O = 1,24 $\mathrm{g} \mathrm{kg}^{-1}$. Granulometria (\%): Areia $=74$, Silte $=11$ e Argila $=15$.

O preparo do solo foi por meio de uma gradagem e abertura manual dos sulcos de plantio com auxílio de enxadas. A adubação da cultura foi realizada com base na análise química do solo e recomendações para a abobrinha italiana de acordo com apontamentos de Trani et al. (2014). No plantio os nutrientes foram distribuídos e incorporados cinco dias antes do transplantio das mudas, aplicando-se $160 \mathrm{~kg} \mathrm{ha}^{-1}$ de $\mathrm{P}_{2} \mathrm{O}_{5} ; 40 \mathrm{~kg}$ ha $^{-1}$ de $\mathrm{K}_{2} \mathrm{O}$ e $30 \mathrm{~kg} \mathrm{ha}^{-1}$ de N. Utilizou-se como fontes de $\mathrm{N}, \mathrm{P}$ e K , uréia, superfosfato simples e cloreto de potássio, respectivamente. Aplicou-se ainda, no plantio, $15 \mathrm{~kg} \mathrm{ha}^{-1}$ de sulfato de zinco, $10 \mathrm{~kg} \mathrm{ha}^{-1}$ de bórax, $10 \mathrm{~kg} \mathrm{ha}^{-1}$ de sulfato de cobre e $0,5 \mathrm{~kg} \mathrm{ha}^{-1}$ de molibdato de amônio. Foram realizadas duas adubações de cobertura com $\mathrm{N}$ e $\mathrm{K}$ aos 20 e 40 dias após o transplantio (DAT) das mudas, e aplicou-se $60 \mathrm{~kg} \mathrm{ha}^{-1}$ de $\mathrm{N}$ e $60 \mathrm{~kg} \mathrm{ha}^{-1}$ de $\mathrm{K}_{2} \mathrm{O}$, metade em cada aplicação, sendo as fontes de $\mathrm{N}$ e $\mathrm{K}$, nitrato de cálcio e cloreto de potássio, respectivamente.

Utilizaram-se no plantio as cultivares de abobrinha italiana dos híbridos Anita F1, Brenda F1, Irit F1, Corona F1 e Caserta, cultivares adquirida junto a empresa Agristar do Brasil linha TopSeed ${ }^{\circledR}$ Premium. As mudas foram produzidas sob ambiente protegido com tela de sombreamento preta $50 \%$, em bandejas multicelulares de 72 células de poliestireno, preenchidas com substrato agrícola comercial "MaxFertil[®" e transplantadas aos 21 dias após a semeadura, quando as plântulas apresentavam dois pares de folhas definitivas.

$\mathrm{O}$ delineamento experimental utilizado foi de blocos casualizados, com quatro repetições e os tratamentos em esquema fatorial $5 \times 2$, compreendendo cinco cultivares de abobrinhas italiana ("Anita F1, Brenda F1, Irit F1, Corona F1 e Caserta") e dois sistemas de cultivo (a pleno sol e sob tela de sombreamento preta a 50\%"). As telas foram instaladas em estruturas tipo "telado" totalmente aberto nas dimensões de 1,5 x 4,0 x $15,0 \mathrm{~m}$ (altura, largura e comprimento). O espaçamento adotado foi de $0,75 \times 0,5 \mathrm{~m}$. As parcelas experimentais constaram de quatro linhas de 3,0 m, em que as duas linhas centrais constituíram a área útil de $3,0 \mathrm{~m}^{2}$, comportando oito plantas centrais, excetuando-se uma planta em cada extremidade das linhas. A polinização ocorreu de forma natural, onde os insetos presentes na área foram os responsáveis pela realização.

Durante a condução do experimento realizaram-se capinas manuais e para o controle de pulgões (Aphis gossypii), broca das cucurbitáceas (Diaphania sp.) e mosca branca (Bemisia sp.) com cinco pulverizações de calda a base de óleo de nim (30\%) realizadas semanalmente a partir dos 15 DAT. A irrigação foi por meio de gotejamento aplicando-se lâminas d'água em torno ente 5 e $10 \mathrm{~mm}$, baseadas na evaporação do tanque classe A.

A colheita dos frutos teve seu início, quando a coloração característica da cultivar "verde escuro ou claro" apresentavam-se brilhantes, tenros, sem fibras, sendo facilmente marcados com a unha, 
indicativo do ponto de colheita. Em seguida foram avaliadas a diâmetro do fruto $(\mathrm{mm})$, comprimento do fruto $(\mathrm{cm})$, massa fresca do fruto ( g fruto $\left.^{-1}\right)$, número de frutos por planta e número de frutos comerciais por planta. As avaliações de massa fresca de fruto, diâmetro de fruto e comprimento de fruto foram realizadas em oito frutos de cada parcela os quais foram pesados e medidos na região mediana do fruto com paquímetro digital.

Os dados obtidos foram submetidos à análise de variância e aplicou-se o teste de Scott-Knott ao nível de 5\% de probabilidade de erro para comparação das médias, e tais análises foram realizadas empregando-se o programa SISVAR 5.4 (Ferreira, 2011).

\section{Resultados e Discussão}

A colheita foi iniciada aos 45 DAT e se prolongou até os 75 DAT e foram realizadas a cada três dias. Não houve interação dos fatores cultivares e sombreamento para as características diâmetro, comprimento e massa média do fruto, verificando-se somente efeito isolado do fator cultivares de abobrinha. Frutos com maior diâmetro foram produzidos pela cultivar Brenda F1 (86,97 mm; +25\%), já Anita F1 $(26,46 \mathrm{~cm}$; $+12 \%)$ e Corona F1 $(25,88 \mathrm{~cm}$; +9\%) produziram frutos com maior comprimento e Brenda F1 $(837,96 \mathrm{~g} ;+40 \%)$ e Corona F1 $(807,30 \mathrm{~g} ;+34 \%)$ frutos com maior massa média, com ganhos expressivos quando comparados aos valores médios obtidos com a cultivar Caserta (Tabela 1).

Tabela 1 Valores médios de diâmetro (DF), comprimento (CF) e massa média (MMF) do fruto de cultivares de abobrinha italiana cultivados sob pleno sol e tela de sombreamento preta $50 \%$

\begin{tabular}{lccc}
\hline Cultivares & $\mathrm{DF}(\mathrm{mm})^{* *}$ & $\mathrm{CF}(\mathrm{cm})^{* *}$ & $\mathrm{MMF}(\mathrm{g})^{* *}$ \\
\hline Anita F1 & $68,40 \mathrm{c}$ & $26,46 \mathrm{a}$ & $637,08 \mathrm{~b}$ \\
Brenda F1 & $86,97 \mathrm{a}$ & $23,70 \mathrm{~b}$ & $837,96 \mathrm{a}$ \\
Irit F1 & $77,56 \mathrm{~b}$ & $22,08 \mathrm{~b}$ & $659,63 \mathrm{~b}$ \\
Corona F1 & $81,96 \mathrm{~b}$ & $25,88 \mathrm{a}$ & $807,30 \mathrm{a}$ \\
Caserta & $69,65 \mathrm{c}$ & $23,70 \mathrm{~b}$ & $600,66 \mathrm{~b}$ \\
\hline \multicolumn{1}{c}{ Ambientes de cultivo } & $\mathrm{DF}(\mathrm{mm})^{\mathrm{ns}}$ & $\mathrm{CF}(\mathrm{cm})^{\mathrm{ns}}$ & $\mathrm{MMF}(\mathrm{g})^{\mathrm{ns}}$ \\
\hline Pleno Sol & $75,88 \mathrm{a}$ & $24,11 \mathrm{a}$ & $721,03 \mathrm{a}$ \\
Tela de sombreamento preta 50\% & $77,49 \mathrm{a}$ & $24,62 \mathrm{a}$ & $696,02 \mathrm{a}$ \\
\hline CV $(\%)$ & 5,55 & 7,61 & 18,51 \\
\hline
\end{tabular}

Médias na coluna seguidas pela mesma letra não diferem entre si pelo teste de Scott-Knott a $5 \%$ de probabilidade. CV: coeficiente de variação. ${ }^{\text {ns }}, \mathrm{e}^{* *}$ : não significativo e significativo a $1 \%$ de probabilidade pelo teste $\mathrm{F}$, respectivamente.

O tamanho dos frutos enquadrou-se dentro dos padrões para cada cultivar. Pode-se inferir que o maior tamanho de fruto de abobrinhas italianas esteja relacionado ao potencial produtivo de cada cultivar e a capacidade de resposta aos fatores essenciais ao crescimento e desenvolvimento dos frutos, tais como água, luz e nutrientes, uma vez que a limitação de água afeta a fase de divisão e expansão celular. Ademais, limitação hídrica associada a pouca disponibilidade luminosa, reduz-se as taxas fotossintéticas, ocasionando menor acúmulo de biomassa e maior competição entre os drenos, levando a obtenção de frutos com menor diâmetro, curtos e leves. Valantin et al. (2006) citam que variações no tamanho final do fruto podem ser interpretadas como consequência de dois processos: a força do dreno durante o período de divisão celular e a taxa de crescimento do fruto durante a expansão celular.
O número de frutos viáveis em plantas de cucurbitáceas, afeta a taxa de crescimento e o seu tamanho final, uma vez que toda expansão celular ocorre após a antese, enquanto que a divisão celular continua acontecer em menor proporção (Queiroga et al., 2008; Valantin et al., 2006). Portanto, quanto maior o número de frutos por planta essa expressão poderá se dá conforme cada cultivar em resposta ao ambiente.

Dessa forma, a produção de frutos de abobrinha italiana com maior tamanho dependerá do potencial produtivo de cada cultivar, do número de células no final da antese e da capacidade dos frutos para drenarem para si, fotoassimilados, estando de acordo com o mencionado por Valantin et al. (2006), que citam no meloeiro um aumento no número de frutos por planta, quando a demanda dos frutos por fotoassimilados se eleva, estabelecendo-se forte competição entre os frutos, e assim, afetando o crescimento dos mesmos. 
Ocorreu interação entre as cultivares e o cultivo sob sombreamento para número de frutos por planta, número de frutos comerciais por planta, produtividade e produtividade comercial (Tabela 2).

O cultivo a pleno sol proporcionou maior número de frutos por planta (3,15 em média) e de frutos comerciais por planta $(2,42$ em média), para todas as cultivares, com exceção da cultivar Irit F1 que não diferiu em relação ao ambiente (Tabela 2).
O cultivo a pleno sol apresentou maior produtividade $(+94 \%)$ e produtividade comercial (+95\%) quando comparado com o cultivo sob tela. A cultivar Brenda F1 apresentou maior produtividade $\left(69,27 \mathrm{t} \quad \mathrm{ha}^{-1} ; \quad+45 \%\right)$ e produtividade comercial $\left(53,34 \mathrm{t} \mathrm{ha}^{-1} ;+45 \%\right)$ em relação a cultivar Caserta $\left(47,90\right.$ e $36,88 \mathrm{t} \mathrm{ha}^{-1}$, respectivamente) cultivada a pleno sol. Já as cultivares Corona F1 $(+21,42 \%$ e $+21,38 \%)$ e Irit $(+16,34 \%$ e $+16,30 \%)$ apresentaram maiores produtividades e produtividade comercial quando cultivada sob tela de sombreamento em relação a cultivar Caserta (Tabela 2).

Tabela 2 Valores médios de número de frutos por planta (NFP), número de frutos comerciais por planta (NFCP), produtividade (PROD) e produtividade comercial (PC) de cultivares de abobrinha italiana cultivadas a pleno sol e sob tela de sombreamento preta $50 \%$

\begin{tabular}{|c|c|c|c|c|c|c|c|c|}
\hline \multirow[b]{2}{*}{ Cultivares } & \multicolumn{3}{|c|}{ NFP** } & \multicolumn{2}{|c|}{ NFCP** } & \multirow{2}{*}{$\begin{array}{c}\begin{array}{c}\text { PROD } \\
\left(\mathrm{t} \mathrm{ha}^{-1}\right)^{* *}\end{array} \\
\text { Tela preta } 50 \%\end{array}$} & \multicolumn{2}{|c|}{$\begin{array}{c}\mathrm{PC} \\
\left(\mathrm{t} \mathrm{ha}^{-1}\right)^{* *}\end{array}$} \\
\hline & $\begin{array}{c}\text { Pleno } \\
\text { Sol }\end{array}$ & $\begin{array}{l}\text { Tela } \\
\text { preta } \\
50 \%\end{array}$ & $\begin{array}{c}\text { Pleno } \\
\text { Sol }\end{array}$ & $\begin{array}{c}\text { Tela } \\
\text { preta } \\
50 \%\end{array}$ & $\begin{array}{l}\text { Pleno } \\
\text { Sol }\end{array}$ & & $\begin{array}{c}\text { Pleno } \\
\text { Sol }\end{array}$ & $\begin{array}{c}\text { Tela preta } \\
50 \%\end{array}$ \\
\hline Anita F1 & $2,7 \mathrm{Aa}$ & $1,5 \mathrm{Bb}$ & $2,1 \mathrm{Aa}$ & $1,1 \mathrm{Bb}$ & $52,22 \mathrm{Ca}$ & $20,25 \mathrm{Cb}$ & $40,20 \mathrm{Ca}$ & $15,60 \mathrm{Cb}$ \\
\hline $\begin{array}{l}\text { Brenda } \\
\text { F1 }\end{array}$ & $3,7 \mathrm{Aa}$ & $1,5 \mathrm{Bb}$ & $2,8 \mathrm{Aa}$ & $1,1 \mathrm{Bb}$ & $69,27 \mathrm{Aa}$ & $22,85 \mathrm{Cb}$ & $53,34 \mathrm{Aa}$ & $17,60 \mathrm{Cb}$ \\
\hline Irit F1 & $3,2 \mathrm{Aa}$ & $3,2 \mathrm{Aa}$ & $2,5 \mathrm{Aa}$ & $2,5 \mathrm{Aa}$ & $59,37 \mathrm{Ba}$ & $35,95 \mathrm{Ab}$ & $47,71 \mathrm{Ba}$ & $27,68 \mathrm{Ab}$ \\
\hline $\begin{array}{l}\text { Corona } \\
\text { F1 }\end{array}$ & $3,0 \mathrm{Aa}$ & $2,0 \mathrm{Bb}$ & $2,3 \mathrm{Aa}$ & $1,5 \mathrm{Bb}$ & $56,75 \mathrm{Ba}$ & $37,52 \mathrm{Ab}$ & $43,70 \mathrm{Ba}$ & $28,89 \mathrm{Ab}$ \\
\hline Caserta & $3,0 \mathrm{Aa}$ & $1,5 \mathrm{Bb}$ & $2,3 \mathrm{Aa}$ & $1,1 \mathrm{Bb}$ & $47,90 \mathrm{Da}$ & $30,90 \mathrm{Bb}$ & $36,88 \mathrm{Da}$ & $23,80 \mathrm{Bb}$ \\
\hline Média & 3,15 & 1,95 & 2,42 & 1,50 & 57,10 & 29,50 & 44,36 & 22,71 \\
\hline $\mathrm{CV}(\%)$ & & 21,79 & & & & 5,91 & & 91 \\
\hline
\end{tabular}

Médias na coluna seguidas pela mesma letra maiúscula e na linha acompanhadas pela mesma letra minúscula, não diferem entre si pelo teste de Scott-Knott a 5\% de probabilidade. CV: coeficiente de variação. ${ }^{\text {ns }}$, e **: não significativo e significativo a $1 \%$ de probabilidade pelo teste $\mathrm{F}$, respectivamente.

Um dos principais fatores responsáveis para a obtenção de altos rendimentos para a abobrinha é a taxa de crescimento e desenvolvimento de frutos na planta, sendo esta relação fortemente influenciada pelas condições climáticas e pelas cultivares.

No cultivo sob tela de sombreamento a menor produtividade pode ser atribuída ao menor número de frutos e, estes, por sua vez, foram afetadas pela menor intensidade luminosa, pois o surgimento de flores femininas ocorre sob alta intensidade luminosa (Ribeiro et al., 2010).

No gênero Curcubita, de modo geral, as plantas são monóicas, com flores masculinas e femininas, sendo as masculinas em número superior (Whitaker e Robinson, 1986) e que em condições de dias curtos, mas com alta intensidade luminosa, as flores femininas são favorecidas. Ribeiro et al. (2010) citam, que não houve formação de frutos de abobrinha sob sombrite $80 \%$ devido não ter ocorrido formação de flores masculinas.

A produtividade da abobrinha foi devido ao maior número de folhas fotossinteticamente ativas, favorecendo o acúmulo individual de massa fresca, e mesmo sob condições de sombreamento, não foram influenciados pela redução das taxas fotossintéticas, mas pelo menor número de flores femininas.

Quando uma cultura está sendo conduzida dentro de uma variação ótima de luminosidade, fato observado neste estudo com sombrite $50 \%$, outros fatores favoráveis, como a fotossíntese é elevada e a quantidade de carboidratos utilizados para o crescimento e desenvolvimento da planta é alta (Edmond et al. 1967). 
Guerra et al. (2017) afirmaram que, incrementos na produtividade de alface cultivado sob tela verde e plástico foram atribuídos ao maior número de folhas fotossinteticamente ativas, favorecendo o acúmulo individual de massa fresca, resultando em alteração nos comprimentos de onda e da temperatura ambiente. Também para Costa et al. (2011), o sombreamento promoveu aumentos na produção total e na massa fresca dos frutos, os quais foram atribuídos à radiação fotossinteticamente ativa, colaborando para a obtenção de temperatura constante no interior do ambiente, favorecendo a concentração do florescimento, além de proporcionar maiores taxas fotossintéticas. Isso ocorre devido à transferência de luz do espectro na faixa de ondas vermelha e vermelha distante ser maior promovendo melhor qualidade de luz e luz difusa e maior desenvolvimento vegetativo, enraizamento e produção (Li, 2006).

Segundo Filgueira (2013) cita que a temperatura ideal para o cultivo de abobrinha ocorre na faixa de 15 a $25^{\circ} \mathrm{C}$, e que o excesso de calor e luminosidade podem comprometer a produção em função do abortamento de flores e a qualidade dos frutos devido a queimaduras pelo sol afetam negativamente $\mathrm{o}$ processo de polinização, e, vingamento de frutos e consequentemente, a produtividade é reduzida.

Contudo, embora exista diferenças entre cultivo a pleno sol e sob telas de sombreamento quanto a produtividade de abobrinha, é válido destacar que nas condições da região Nordeste, principalmente nos meses de Setembro a Dezembro com a intensa insolação e a baixa umidade relativa do ar, o cultivo sob tela pode minimizar esses efeitos negativos do ambiente sobre os frutos. Nesse sentido, as telas de sombreamento reduzirão o excesso de radiação solar, sem causar prejuízos a formação de flores femininas, garantindo a produção de frutos e evitando as queimaduras que ocorrem em frutos que são cultivados a pleno sol, garantindo assim, suas qualidades visuais, nutricionais e sensoriais.

\section{Conclusões}

No cultivo de outono, na região do Nordeste brasileiro, a abobrinha deve ser produzida a pleno sol por proporcionar maior produtividade;

Entre as cultivares de abobrinhas italianas, Anita F1, Brenda F1, Irit F1, Corona F1 e Caserta, recomenda-se Brenda $\mathrm{F} 1$ pois possui tanto maior produtividade total quanto maior produtividade comercial no ciclo de outono no Nordeste brasileiro.

\section{Agradecimentos}

A empresa Agristar do Brasil pela doação das sementes das cultivares de abobrinha italiana.

\section{Referências}

Anglés, M. Control climático y ciclo de cultivo. Horticultura, v. 152, p. 1-7, 2001.

Costa, R. C.; Calvete, E. O.; Reginatto, F. H.; Cecchetti, D.; LOSS, J. T.; Rambo, A.; Tessaro, F. Telas de sombreamento na produção de morangueiro em ambiente protegido. Horticultura Brasileira, v. 1, n. 29, p. 98-102, 2011. http://dx.doi.org/10.1590/S0102-05362011000100016

Duarte, T. S.; Peil, R. M. N. Relações fonte:dreno e crescimento vegetativo do meloeiro. Horticultura Brasileira, v. 28, n. 3, p. 271276, 2010. http://dx.doi.org/10.1590/S010205362010000300005

Edmond, J. B.; Senn, T. L.; Andrews, E. S. Princípios de horticultura. México: Continental, p. 119-134. 1967.

Ferreira, D. F. Sisvar: a computer statistical analysis system. Ciência e Agrotecnologia, v. 35, p. 1039-1042, 2011. https://doi.org/10.1590/S1413-70542011000600001

Filgueira, F. A. R. Novo Manual de Olericultura: agrotecnologia moderna na produção e comercialização de hortaliças. 3 . ed. ver. e ampl. - Viçosa, MG: Ed. UFV, 2013. $421 \mathrm{p}$.

Guerra, A. M. N. M.; Costa, A. C. M.; Tavares, P. R. F. Atividade fotossintética e produtividade de alface cultivada sob sombreamento. Agropecuária Técnica, v. 38, n. 3, p. 125-132, 2017. -https://doi.org/10.25066/agrotec.v38i3.29246

Huertas, L. Control ambiental em el vivero. Horticultura Internacional, n. extra: p. 77-84, 2006.

IBGE - Instituto Brasileiro de Geografia e Estatística. 2017. Número de estabelecimentos agropecuários e Quantidade produzida, por produtos da horticultura - resultados preliminares 2018. Disponível em: < https://www.ibge.gov.br/estatisticasnovoportal/economicas/agricultura-e-pecuaria/21814- 
2017-censo-agropecuario.html?=\&t=resultados >. Acesso em 28 de junho de 2020.

INMET. 2018. BDMEP Dados históricos. Disponível em: < http://www.inmet.gov.br/portal/index.php?r=bdmep/bdm ep >. Acessado em 19 de janeiro de 2020.

Li, J. C. Uso de mallas en invernaderos. Horticultura Internacional, n. extra, p. 86-91, 2006.

Madeira, N. R.; Amaro, G. B. Exigências climáticas e ecofisiológicas. In: Abóboras e morangas do plantio à colheita. Ed. Nick, C; Borém, A. Viçosa: Editora UFV. 203p. 2017.

Queiroga, R. C. F.; Puiatti, M.; Fontes, P. C. R.; Cecon, P. R. 2008. Produtividade e qualidade de frutos de meloeiro variando número de frutos e de folhas por planta. Horticultura Brasileira, v. 26, n. 2, p. 209-215, 2008. https://doi.org/10.1590/S0102-05362008000200016

Ribeiro, D. H.; Arfinengo, A. R. E.; Fogaca, I. S.; Benites, F. J.; Pasin, L. A. A. P. Interferência de diferentes condições de luminosidade nos Padrões fenológicos de Cucurbita pepo L. e Solanum gilo. Revista Científica Eletrônica de Agronomia, v. 17, n. 1, p. 1-6, 2010. http://faef.revista.inf.br/imagens_arquivos/arquivos_desta que/atSnLvlsInFQsVu_2013-5-10-16-18-53.pdf

Santi, A.; Scaramuzza, W. L. M. P.; Soares, D. M. J.; Scaramuzza, J. F.; Dallacort, R.; Krause, W.; Tieppo, R. C. Desempenho e orientação do crescimento do pepino japonês em ambiente protegido. Horticultura Brasileira, v. 31, n. 4, p. 649-653, 2013. http://dx.doi.org/10.1590/S010205362013000400023

Shahak, Y.; Gussakovsky, E. E.; Cohen, Y.; Lurie, S.; Stern, R.; Kfir, S.; Naor, A.; Atzmon, I.; Doron, I.; Greenblat-Avron, Y. ColorNets: a new approach for light manipulation in fruit trees. Acta Horticulturae, v. 636, p. 609-616, 2004.

https://doi.org/10.17660/ActaHortic.2004.636.76

Trani, P. E.; Passos, F. A.; Araújo, H. S. Calagem e adubação da abobrinha italiana (de moita) (Curcubita pepo), abóbora brasileira (Cucurbita moschata), moranga (Cucurbita máxima) e abóbora japonesa (híbrida). 2014. Disponível em:

http://www.iac.sp.gov.br/imagem_informacoestecn ologicas/96.pdf >. Acesso em: 15 de janeiro de 2020.

Valantin, M.; Vaissiere, B. E.; Gary, C.; Robin, P. Source-sink balance affects reproductive development and fruit quality in cantaloupe melon. Journal of Horticultural Science \& Biotechnology, v. 81, n. 1, p. 105-117, 2006. https://doi.org/10.1080/14620316.2006.11512036

Whitaker, T. W.; Robinson, R. W. Squash breeding. In: Basset, M. J. (Ed.) Breeding vegetable crops. West port: Avi Publishing, 1986. Cap.6, p.209-242. 